\title{
Hot-hole-induced interface states build-up on deep-submicrometer LDD nMOSFETs
}

\author{
J. M. Rafí and F. Campabadal \\ Institut de Microelectrònica de Barcelona, CNM-CSIC \\ Campus UAB, 08193 Bellaterra (Barcelona), Spain
}

\begin{abstract}
The hot-carrier degradation of deep-submicrometer LDD nMOSFETs under different gate-stress regimes is analysed by means of current-voltage and charge pumping characteristics. Interface state generation is found to arise as the major mechanism responsible for device degradation in the whole range of gate regimes studied.

The effects of Short Electron and Hole Injection phases on hot-carrier-stressed devices are also analysed. Although SEI phases are found to be an efficient tool for revealing part of the damage generated in stresses at low gate voltages, the performance of a first SHI phase after stress at high gate bias $\left(\mathrm{V}_{\mathrm{g}}>\mathrm{V}_{\mathrm{d}} / 2\right)$ results in a significant additional degradation of the devices. This enhanced degradation is attributed to a sudden interface states build-up occurring near the Si/spacer interface only under the first hot-hole injection condition.
\end{abstract}

\section{Introduction}

The problems associated with hot carriers have been recognised as one of the major constraints in $\mathrm{Si}$ MOS scaling. Hot carriers are generated near the drain junction by the high electric field and may attain sufficient energy to overcome the $\mathrm{Si}-\mathrm{SiO}_{2}$ barrier, causing damage in this region, which leads to a gradual degradation of the transistor currentvoltage characteristics. The damage is commonly believed to result in interface-state generation at the $\mathrm{Si}-\mathrm{SiO}_{2}$ interface [1] and charge trapping in the gate oxide [2]. However, the specific particularities involving the different gate regimes and the drain architectures make physical understanding of hotcarrier degradation a rather complex subject.

In the past years, Short Electron Injection phases (SEI) carried out at $\mathrm{V}_{\mathrm{g}}=\mathrm{V}_{\mathrm{d}}$ have been shown to be a useful technique to reveal a significant amount of the damage generated under low gate bias stress, which cannot be fully detected by means of simple drain current $\left(\mathrm{I}_{\mathrm{d}}\right)$ vs. gate voltage $\left(\mathrm{V}_{\mathrm{g}}\right)$ curves. The impact of this additional damage is specially important for lifetime evaluation and $\mathrm{AC}$ operation $[3,4]$. On the other hand, the performance of a subsequent Short Hole Injection phase (SHI) at high $\mathrm{V}_{\mathrm{d}}$ and $\mathrm{V}_{\mathrm{g}}$ near threshold voltage, $\mathrm{V}_{\text {th }}$, was thought to merely return the $I_{d}$ vs. $V_{g}$ curves towards the poststress situation, without causing any significant additional degradation of the devices $[3,5,6]$. In the case of longer hole-injection periods, a progressive generation of interface traps was observed $[7,8]$.

In this paper, the effects of SEI and SHI phases on deep-submicrometer hot-carrier-stressed LDD nMOSFETs are analysed and the effect of a first SHI phase after stress at high gate bias $\left(V_{\mathrm{g}}>\mathrm{V}_{\mathrm{d}} / 2\right)$ is shown to result in a significant additional degradation of the devices. This phenomenon occurs suddenly and it is attributed to an interface states build-up occurring at the $\mathrm{Si} /$ spacer interface only under the first hot-hole injection condition. 


\section{Experimental procedure and devices}

The n-channel MOSFET devices used in this study were fabricated using a conventional $0.35 \mu \mathrm{m}-$ CMOS technology with Lightly Doped Drain (LDD) technology. The specific dimensions of the transistors under study are: $\mathrm{L}_{\text {poly }}=0.45 \mu \mathrm{m}, \mathrm{W}=40 \mu \mathrm{m}$, an $8 \mathrm{~nm}$-thick $\mathrm{SiO}_{2}$ gate dielectric and $0.12 \mu \mathrm{m}$-long oxide spacers.

Previous to the study of device degradation, an electrical characterisation was carried out, determining the most fundamental parameters, such as threshold voltage $\left(\mathrm{V}_{\mathrm{th}}=0.55 \mathrm{~V}\right)$ and saturation drain current $\left(\mathrm{I}_{\mathrm{dsat}}\left(@ \mathrm{~V}_{\mathrm{g}}=\mathrm{V}_{\mathrm{d}}=3.3 \mathrm{~V}\right)=375 \mu \mathrm{A} / \mu \mathrm{m}\right)$. In addition, the most representative aspects of drain and substrate characteristics were evaluated, and the gate voltage condition leading to maximum substrate current was determined to be at $\mathrm{V}_{\mathrm{g}} \approx \mathrm{V}_{\mathrm{d}} / 2-0.75 \mathrm{~V}$.

For the hot-carrier degradation analysis, the transistors were stressed at constant-voltage stress condition, under a fixed drain bias $\left(\mathrm{V}_{\mathrm{d}}=6 \mathrm{~V}\right)$ and with different gate voltages $\left(\mathrm{V}_{\mathrm{g}}\right)$ above and below maximum substrate current condition, for periods up to $50000 \mathrm{~s}$. In order to evaluate device degradation, $\mathrm{I}_{\mathrm{d}}$ vs. $\mathrm{V}_{\mathrm{g}}$ at $\mathrm{V}_{\mathrm{d}}=0.1 \mathrm{~V}$ together with charge pumping characteristics were regularly measured between different stress phases.

Threshold voltage $\left(\mathrm{V}_{\mathrm{th}}\right)$, defined as the gate voltage for a fixed drain current of $0.01 \cdot \mathrm{W} / \mathrm{L} \mu \mathrm{A}$, maximum transconductance $\left(\mathrm{G}_{\operatorname{mmax}}\right)$ and linear drain current measured at $\mathrm{V}_{\mathrm{g}}=3.3 \mathrm{~V}\left(\mathrm{I}_{\mathrm{dlin}}\right)$ were the primary degradation monitors, all of them obtained from the $I_{d}$ vs. $V_{g}$ curves. The charge pumping current increase at a frequency of $250 \mathrm{kHz}, \Delta \mathrm{I}_{\mathrm{cp}}$, was adopted as the indicator for interface state generation.

The fully automated experimental set-up used for both, the electrical characterisation and the stress experiments, consisted of an HP 4142B modular DC source/monitor, an HP 4085A switching matrix and a Wentworth automatic wafer prober. A Keithley 3940 pulse generator and a 485 picoammeter were used for the charge pumping measurements. All experiments were carried out in a light-proof and electrically shielded probe station.

\section{Results and discussion}

\subsection{Device degradation and SEI phases}

During the aging experiments, no significative threshold voltage shifts were registered, whereas

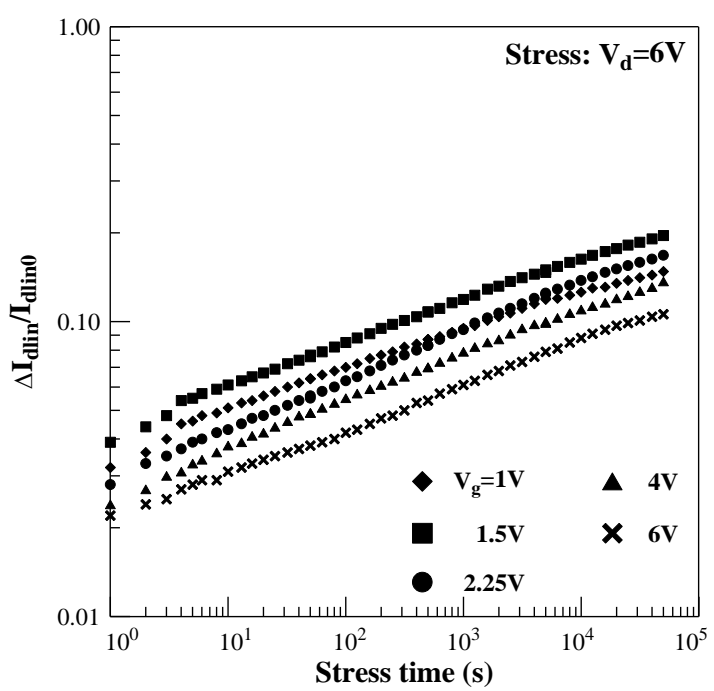

Fig. 1. Linear drain current degradation $\left(\Delta \mathrm{I}_{\mathrm{dlin}} / \mathrm{I}_{\mathrm{dlin} 0}\right)$ as a function of stress time for different devices stressed at $\mathrm{V}_{\mathrm{d}}=6 \mathrm{~V}$ and $\mathrm{V}_{\mathrm{g}}=1,1.5,2.25,4$ and $6 \mathrm{~V}$.

device degradation was evaluated through the evolution of $G_{\operatorname{mmax}}$ and $I_{\text {dlin. }}$. In Fig. 1, the degradation of $\mathrm{I}_{\mathrm{dlin}}$ corresponding to five different devices stressed at a fixed drain voltage $\left(\mathrm{V}_{\mathrm{d}}=6 \mathrm{~V}\right)$ and various gate biases is shown as a function of stress time. It is found that $\Delta \mathrm{I}_{\mathrm{dlin}} / \mathrm{I}_{\mathrm{dlin} 0}$ (a similar result is obtained for $\Delta G_{\operatorname{mmax}} / G_{\operatorname{mmax} 0}$ ) can be approached by the typical power-law dependency proposed by Takeda et al. [9]:

$$
I_{d l i n} / I_{d l i n 0}=A t^{n}
$$

being $\mathrm{I}_{\mathrm{dlin} 0}$ the linear drain current of the virgin device, A the magnitude of the degradation parameter, $\mathrm{n}$ the gradient of the time power law and $\mathrm{t}$ the stress time.

Different values for A, related to lateral electric field and impact ionisation population, are found for the various $\mathrm{V}_{\mathrm{g}}$-stress conditions. However, no significant differences in degradation slope (n) are registered for these samples under the given $\mathrm{V}_{\mathrm{d}}$ stress condition, indicating that a major dominant mechanism, common for the whole $\mathrm{V}_{\mathrm{g}}$-stress range, is responsible for the degradation

A close examination of these results, together with the corresponding charge pumping characterisation, is given in Fig. 2, for various aging periods corresponding to the different $\mathrm{V}_{\mathrm{g}}$-stress experiments of Fig. 1. In Fig. 2(a) it is shown that the condition giving rise to maximum hot-carrier 


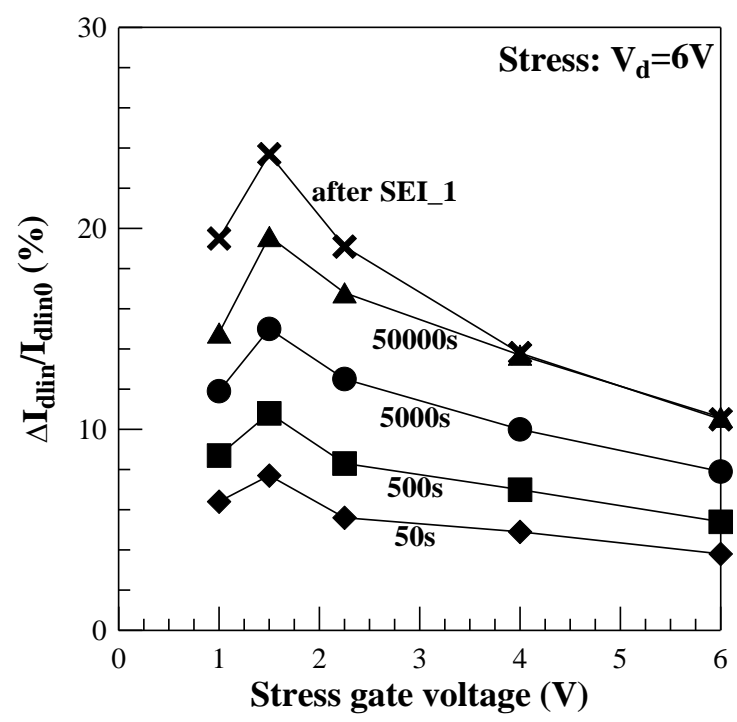

(a)

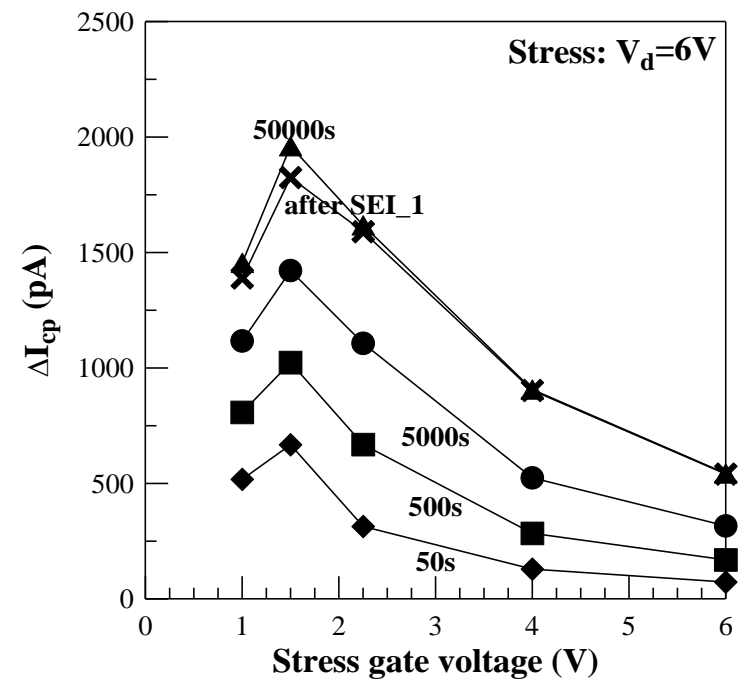

(b)

Fig. 2. (a) Linear drain current degradation and (b) charge pumping current increase as a function of stress gate voltage for different aging times and after a SEI phase.

degradation corresponds to $\mathrm{V}_{\mathrm{g}}=1.5 \mathrm{~V}$, whereas $\mathrm{V}_{\mathrm{g}}=\mathrm{V}_{\mathrm{d}}$ correspond to the less harmful stress regime. When comparing the drain current degradation with the charge pumping current increase, shown in Fig. 2(b), a high correspondence is found between both parameters, indicating a close relationship between device degradation and interface state generation for all $\mathrm{V}_{\mathrm{g}}$ conditions.

In order to better evaluate the real amount of damage created under the different gate stress conditions, SEI phases of $10 \mathrm{~s}$ at $\mathrm{V}_{\mathrm{g}}=\mathrm{V}_{\mathrm{d}}=6 \mathrm{~V}$ were performed after each aging experiment. $\mathrm{I}_{\mathrm{dlin}}$ degradation results after performing a first SEI phase (SEI_1) subsequent to 50000s stress are also given in Fig. 2(a). It is shown that only for low gate voltage stresses $\left(\mathrm{V}_{\mathrm{g}}<2.25 \mathrm{~V}\right) \Delta \mathrm{I}_{\text {dlin }} / \mathrm{I}_{\text {dlino }}$ substantially increases, whereas for higher $\mathrm{V}_{\mathrm{g}}$ stresses, no additional degradation of $I_{\text {dlin }}$ is observed after the SEI phase.

Discussion about the nature of the SEIenhanced degradation has led in the past to remarkable opposite conclusions between different research groups $[10,11]$.

It is well accepted that both, gate oxide hole trapping and generation of a certain amount of fast interface traps take place during hot carrier stress at low gate biases $\left(\mathrm{V}_{\mathrm{g}}<\mathrm{V}_{\mathrm{d}} / 2\right)$. However, an important number of studies using different characterisation techniques ( $\mathrm{I}-\mathrm{V}$ characteristics, charge pumping, floating gate currents [6] and electron detrapping experiments [4]) concluded that a third degradation mechanism dominating interface state generation effects should be considered. This is neutral (acceptor-like) oxide traps generation [2,3]. These neutral traps, generated during stress at low gate biases, would be only revealed after filling by electrons (i.e. at the voltage condition $\mathrm{V}_{\mathrm{g}}=\mathrm{V}_{\mathrm{d}}$ ). This would easily explain the enhanced hot-carrier degradation observed after performing the SEI phase.

On the other hand, a second group of studies concluded that there was neither evidence nor need for invoking the generation of neutral traps during hole-injection conditions. Consequently, they explained similar or even the same results in terms of a model where hole trapping and interface state generation were the primary degradation mechanisms [10]. According to this model, the important amount of interface states generated during a low gate voltage stress becomes partially masked by the effect of trapped holes also generated during the same stress. Thus, the enhanced hotcarrier degradation observed when a SEI phase is performed is explained as being the result of neutralisation of previously trapped holes by the injection of electrons at the $\mathrm{V}_{\mathrm{g}}=\mathrm{V}_{\mathrm{d}}$ condition.

In order to better investigate the nature of the SEI-enhanced degradation, charge pumping characteristics were also measured after the SEI phases. The registered values for $\Delta \mathrm{I}_{\mathrm{cp}}$ are also shown in Fig. 2(b). As it was presumable by its low duration and by the $\mathrm{V}_{\mathrm{g}}=\mathrm{V}_{\mathrm{d}}$ condition, $\Delta \mathrm{I}_{\mathrm{cp}}$ results 
show that no additional interface state generation takes place during the SEI phase. Thus, the SEIenhanced I-V degradation must be attributed to a manifestation of the already existing damage.

An important proof for the dominant role of interface state generation in hot-carrier degradation experiments for the whole $\mathrm{V}_{\mathrm{g}}$-stress range analysed is given by Fig. 3 results. In this figure, $I_{d l i n}$ degradation values are plotted against their counterparts corresponding to $\Delta \mathrm{I}_{\mathrm{cp}}$. Two linear relationships can be inferred between this pair of variables, showing that charge pumping current increase due to interface state generation arises as an excellent monitor of hot-carrier degradation for the whole $\mathrm{V}_{\mathrm{g}}$-stress range. In addition, the marked $\Delta \mathrm{I}_{\mathrm{dlin}} / \mathrm{I}_{\mathrm{dlin} 0}$ shift existing between lines corresponding to low gate voltage stresses $\left(\mathrm{V}_{\mathrm{g}}<2.25 \mathrm{~V}\right)$ and high gate voltage ones is experimentally overcome by carrying out a SEI phase.

From Fig. 3, the fact that for equal $\Delta \mathrm{I}_{\mathrm{cp}}$ we have equal $\Delta \mathrm{I}_{\mathrm{dlin}} / \mathrm{I}_{\mathrm{dlin} 0}$ is interpreted as a clear proof that device degradation is mainly determined by the total amount of fast interface traps. Furthermore, the fact that a SEI phase allows to overcome the $\Delta \mathrm{I}_{\mathrm{dlin}} / \mathrm{I}_{\mathrm{dlin} 0}$ shift existing between low and high gate voltage results, is the result of a hole trapping neutralisation process. In this way, if acceptor-like oxide traps were responsible for the enhanced hotcarrier degradation observed after a SEI phase, then, for a given $\Delta \mathrm{I}_{\mathrm{cp}}$ value, $\Delta \mathrm{I}_{\mathrm{dlin}} / \mathrm{I}_{\mathrm{dlin} 0}$ should be much larger than the corresponding to the established $\Delta \mathrm{I}_{\mathrm{dlin}} / \mathrm{I}_{\mathrm{dlin} 0}$ vs. $\Delta \mathrm{I}_{\mathrm{cp}}$ relationship. As a consequence, our results show, as in [8], that low gate voltage stresses are responsible for an important hole trapping and this partly masks interface states degradation effects. Only when those trapped holes are removed by the application of a SEI phase, are the interface states degradation effects revealed in their entirety.

\subsection{Device degradation and SHI phases}

In order to further investigate the degradation mechanisms discussed in the above paragraphs and the nature of the damage created under the different gate stress conditions, $\mathrm{SHI}$ phases of $50 \mathrm{~s}$ at $\mathrm{V}_{\mathrm{d}}=6 \mathrm{~V}$ and $\mathrm{V}_{\mathrm{g}}=1 \mathrm{~V}$ were subsequently performed after the SEI phases. It was expected that hole injection would essentially result in positive charge trapping, leading to a significant compensation of device degradation for all previously stressed devices.

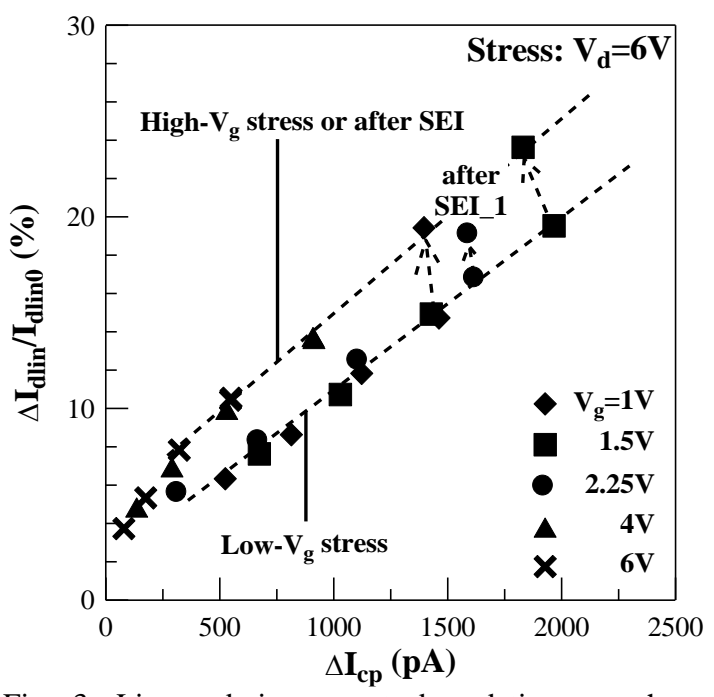

Fig. 3. Linear drain current degradation vs. charge pumping current increase for different $\mathrm{V}_{\mathrm{g}}$-stress conditions and after a SEI phase.

In Fig. 4(a), $I_{\text {dlin }}$ degradation results after a first SHI phase (SHI_1) subsequent to SEI_1 phase are shown. Partial recovery of $\mathrm{I}_{\text {dlin }}$ degradation with respect to after-SEI_1 situation is clearly appreciated for those devices which were previously subjected to low $\mathrm{V}_{\mathrm{g}}$ stresses. However, as the recovery is lower or even null for the devices stressed at $\mathrm{V}_{\mathrm{g}}=4$ and $6 \mathrm{~V}$, an additional explanation should be given for these two latter cases.

Charge pumping current increase results after SHI_1 phase are shown in Fig. 4(b). There is no additional device degradation for those devices prestressed at low gate voltages. In addition, SHI_1 phase is responsible for an important interface state generation in the devices stressed at $\mathrm{V}_{\mathrm{g}}=4$ and $6 \mathrm{~V}$. This interface states build-up during SHI_1 phase is believed to give rise to the low or even null $\Delta \mathrm{I}_{\text {dlin }} / \mathrm{I}_{\text {dlin0 }}$ recovery registered for these two cases after the SEI_1 phase. When a subsequent SEI phase is performed (SEI_2), enhanced $\mathrm{I}_{\text {dlin }}$ degradation is observed for $\mathrm{V}_{\mathrm{g}}=4$ and $6 \mathrm{~V}$ pre-stressed devices. This enhanced $I_{\text {dlin }}$ degradation, which was not observed after the SEI_1 phase, is now registered thanks to both, hole trapping and interface state generation taking place during SHI_1 phase. In this way, while interface state generation is responsible for the higher $\Delta \mathrm{I}_{\mathrm{dlin}} / \mathrm{I}_{\mathrm{dlin} 0}$ value registered after SEI_2 phase, trapped holes during SHI_1 phase are responsible for the masking effect. 


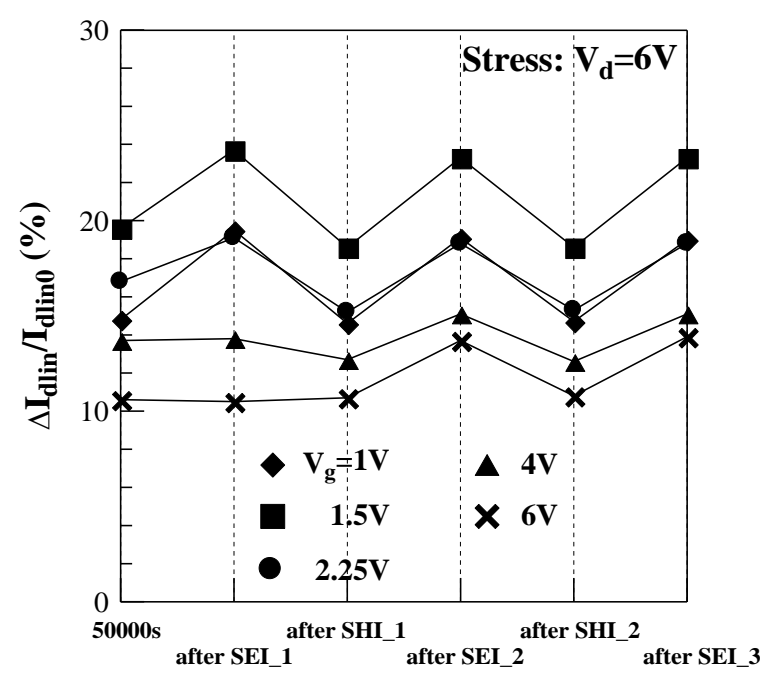

(a)

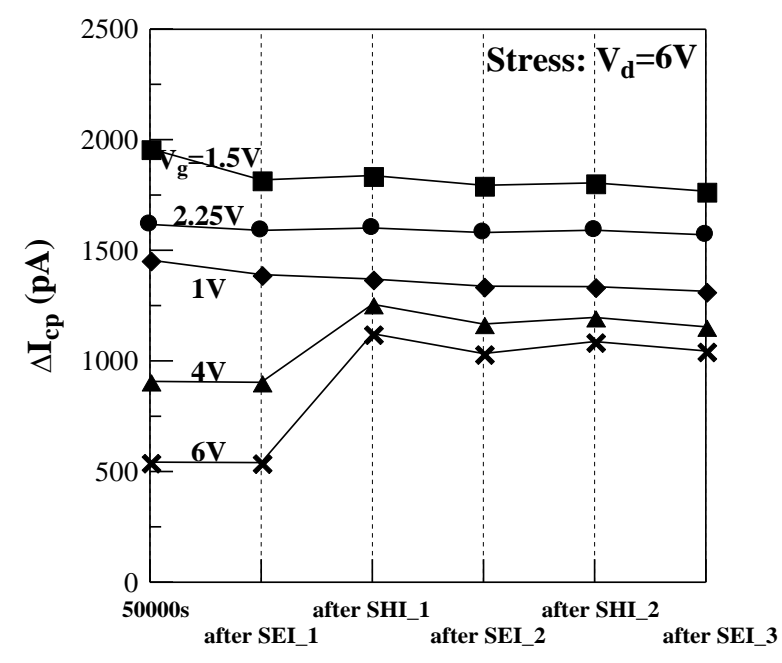

(b)

Fig. 4. (a) Linear drain current degradation and (b) charge pumping current increase at the end of different $\mathrm{V}_{\mathrm{g}}$-stresses (50000s) and after subsequent SEI and SHI phases.

The experiment can then be continued. As the time and voltage conditions for SHI_1 and SHI_2 phases are the same, it was expected some interface state generation to occur during the SHI_2 phase. However, no additional damage is generated during subsequent SHI phases (SHI_2) (see $\Delta \mathrm{I}_{\mathrm{cp}}$ results in Fig. 4(b)), only the masking and neutralisation effect is observed. Each time holes are injected (SHI_2), interface traps effects are partly masked by trapped holes and $\Delta \mathrm{I}_{\text {dlin }} / \mathrm{I}_{\mathrm{dlin} 0}$ decreases. Subsequent electron injection (SEI_3) neutralises trapped holes and $\Delta \mathrm{I}_{\mathrm{dlin}} / \mathrm{I}_{\mathrm{dlin} 0}$ increases again.

The observed controversy concerning the damage build-up registered during SHI phases shows that only the first hot-hole injection phase is responsible for the generation of a significant amount of interface traps. This can be the result of the degradation of a localised region which was not previously damaged under the high gate bias conditions. Taking into account the fact that the hole injection probability to the gate is higher under the lowest gate bias regimes and that it increases towards the drain edge of the channel [2], we associate the interface states spring-up to the effect of injected holes in the proximity of the Si/spacer oxide interface. Once the interface traps build-up has occurred in that zone, it never again contributes to device degradation in such a sudden way.

\section{Conclusions}

In this paper, the hot-carrier degradation of deep-submicrometer LDD devices under different gate-stress regimes has been analysed. Interface state generation has been found to arise as the major mechanism responsible for device degradation in the whole range of experimental conditions analysed. By using SEI phases, it has been possible to reveal a significant amount of the damage generated under low gate bias stresses, which was masked by the effect of the trapped holes generated under this condition.

The effect of a subsequent SHI phase after stress at high gate bias $\left(V_{\mathrm{g}}>\mathrm{V}_{\mathrm{d}} / 2\right)$ has been proved to result in a significant additional degradation of the devices. This enhanced degradation is attributed to a sudden interface states build-up occurring near the $\mathrm{Si} /$ spacer interface only under the first hot-hole injection condition.

\section{Acknowledgements}

This work has been partially supported by the ESPRIT ADEQUAT II project No. 8002 and by the Comisión Interministerial de Ciencia y Tecnología (CICYT), under project TIC95-1278-CE. The samples were supplied by GRESSI-CNET (Meylan, France). 


\section{References}

[1] $\mathrm{Hu}$ C., et al. Hot-electron-induced MOSFET degradation - model, monitor and improvement. IEEE Trans. Electron Devices. ED-32 (1985) 375385.

[2] Tsuchiya T., Kobayashi T. and Nakajima S. Hotcarrier-injected oxide region and hot-electron trapping as the main cause in $\mathrm{Si}$ nMOSFET degradation. IEEE Trans. Electron Devices. ED-34 (1987) 386-391.

[3] Bravaix A. and Vuillaume D. Analysis of the hotcarrier degradation of deep-submicrometer largeangle-tilt-implanted drain (LATID) MOSFETs. Solid-St. Electron. v. 41 (1997) 1293-1302.

[4] Weber W., et al. Hot-hole-induced negative oxide charges in n-MOSFET's. IEEE Trans. Electron Devices. v. 42 (1995) 1473-1480.

[5] Tsuchiya T. Trapped-electron and generated interface-trap effects in hot-electron-induced MOSFET degradation. IEEE Trans. Electron Devices. ED-34 (1987) 2291-2296.

[6] Vuillaume D., et al. A coupled study by floatinggate and charge-pumping techniques of hot-carrierinduced defects in submicrometer LDD nMOSFET's. IEEE Trans. Electron Devices. v. 40 (1993) 773-781.

[7] Bellens R., et al. Hot-carrier effects in n-channel MOS transistors under alternating stress conditions. IEEE Electron Dev. Lett. v. 9 (1988) 232-234.

[8] Heremans P., et al. Consistent model for the hotcarrier degradation in $\mathrm{n}$-channel and $\mathrm{p}$-channel MOSFET's. IEEE Trans. Electron Devices. v. 35 (1988) 2194-2209.

[9] Takeda E. and Suzuki N. An empirical model for device degradation due to hot-carrier injection. IEEE Electron Dev. Lett. EDL-4 (1983) 111-113.

[10] Heremans P., et al. and Doyle B. S., et al. Comment on the generation and characterization of electron and hole traps created by hole injection during low gate voltage hot-carrier stressing of n-MOS transistors. IEEE Trans. Electron Devices. v. 39 (1992) 458-464.

[11] Vuillaume D., et al. and Weber W., et al. Comment on hot-hole-induced negative oxide charges in nMOSFET's. IEEE Trans. Electron Devices. v. 43 (1996) 1473-1477. 